\title{
A Hot Saturn Near (but Unassociated with) the Open Cluster NGC 1817
}

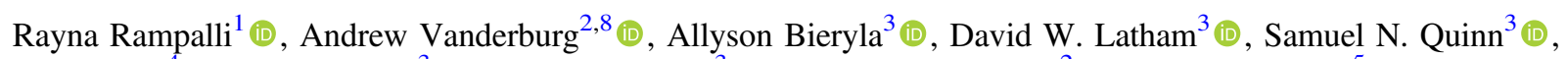 \\ Christoph Baranec ${ }^{4}$ (D), Perry Berlind ${ }^{3}$, Michael L. Calkins ${ }^{3}$ (D), William D. Cochran ${ }^{2}$ (D), Dmitry A. Duev ${ }^{5}$ (D) Michael Endl ${ }^{2}$ (D), \\ Gilbert A. Esquerdo ${ }^{3}$, Rebecca Jensen-Clem ${ }^{6}$ (D), Nicholas M. Law ${ }^{7}$ (D), Andrew W. Mayo ${ }^{6}$ (D), Reed Riddle ${ }^{5}$ (iD), and \\ Maïssa Salama ${ }^{4}$ (iD \\ ${ }^{1}$ Department of Astronomy, Columbia University, 550 West 120th Street, New York, NY 10027, USA; rmr2196@ columbia.edu \\ ${ }^{2}$ Department of Astronomy, The University of Texas at Austin, Austin, TX 78712, USA \\ ${ }^{3}$ Center for Astrophysics|Harvard \& Smithsonian, 60 Garden Street, Cambridge, MA 02138, USA \\ ${ }^{4}$ Institute for Astronomy, University of Hawai 'i at Mānoa, Hilo, HI 96720-2700, USA \\ ${ }^{5}$ Department of Astronomy, California Institute of Technology, 1200 East California Boulevard, Pasadena, CA 91101, USA \\ ${ }^{6}$ Astronomy Department, University of California Berkeley, Berkeley, CA 94720-3411, USA \\ ${ }^{7}$ Department of Physics and Astronomy, University of North Carolina at Chapel Hill, Chapel Hill, NC 27599-3255, USA \\ Received 2019 April 23; revised 2019 June 3; accepted 2019 June 4; published 2019 July 17
}

\begin{abstract}
We report on the discovery of a hot Saturn-sized planet $\left(9.916 \pm 0.985 R_{\oplus}\right)$ around a late F-star, K2-308, observed in Campaign 13 of the $K 2$ mission. We began studying this planet candidate because prior to the release of Gaia DR2, the host star was thought to have been a member $(\geqslant 90 \%$ membership probability) of the $\approx 1$ Gyr open cluster NGC 1817 based on its kinematics and photometric distance. We identify the host star (among three stars within the $K 2$ photometric aperture) using seeing-limited photometry and rule out false-positive scenarios using adaptive optics imaging and radial velocity observations. We statistically validate $\mathrm{K} 2-308$ by calculating a false-positive probability rate of $0.01 \%$. However, we also show using new kinematic measurements provided by Gaia DR2 and our measured radial velocity of the system that K2-308 is unassociated with the cluster NGC 1817. Therefore, the long running search for a giant transiting planet in an open cluster remains fruitless. Finally, we note that our use of seeing-limited photometry is a good demonstration of similar techniques that are already being used to follow up Transiting Exoplanet Survey Satellite (TESS) planet candidates, especially in crowded regions.
\end{abstract}

Key words: planetary systems - planets and satellites: detection - stars: individual (K2-308)

\section{Introduction}

Open clusters have long served as benchmarks for studying stellar, dynamical, and most recently, planet evolution due to their homogeneous nature. These populations provide a sample of stars of approximately the same age, distance, and metallicity over a large range in mass. As a result, when studying how exoplanets depend on their host stellar properties, clusters are ideal environments to target.

Additionally, since open clusters are often made up of young stellar populations, studying planets in open clusters is imperative for characterizing planetary evolution since the most formative time for planets is thought to be in the first gigayear (e.g., Mann et al. 2010; Lopez et al. 2012). For example, on this timescale, the increased stellar rotation and magnetic activity lead to an excess of X-ray and ultraviolet emission that could erode atmospheres of close-in planets (Lammer et al. 2014). Comparing planets found in the younger stellar populations to those found around older field stars could illuminate just exactly how planetary systems evolve.

There have been a number of planet surveys targeting clusters, and a handful of planets have been discovered. Through radial velocity (RV) surveys, several hot Jupiters were discovered in the Hyades and Praesepe, both around 650 Myr in age (Sato et al. 2007; Quinn et al. 2012, 2014). The $K 2$ mission (Howell et al. 2014) has observed a number of clusters (including the Hyades, Praesepe, and others) and has yielded close-in, Neptune-sized, and smaller planet

\footnotetext{
${ }^{8}$ NASA Sagan Fellow.
}

detections (e.g., Crossfield et al. 2015; Mann et al. 2016; Pepper et al. 2017; Curtis et al. 2018; Vanderburg et al. 2018). However, there have not been any transiting giant planets found around clusters, most likely because hot Jupiters are intrinsically rare (van Saders \& Gaudi 2011). The largest transiting planets in clusters found to date are Neptune-sized planets orbiting stars in NGC 6811 (Meibom et al. 2013).

NGC 1817 is an open cluster observed by $K 2$ in its thirteenth campaign. Its age is between 0.8 and $1.2 \mathrm{Gyr}$, similar to that of both the Hyades and Praesepe with a subsolar metallicity of $[\mathrm{Fe} / \mathrm{H}]=-0.40 \mathrm{dex}$ (Donati et al. 2014). Located in the constellation of Taurus, $1.7 \mathrm{kpc}$ from Earth, it is $9.6 \mathrm{kpc}$ from the Galactic center with a proper motion of 0.485 R.A., -0.89 decl. mas $\mathrm{yr}^{-1}$, and an $\mathrm{RV}$ of $65.3 \pm 0.1 \mathrm{~km} \mathrm{~s}^{-1}$ (BalaguerNúñez et al. 2004; Cantat-Gaudin et al. 2018).

In this paper, we initially identified a giant planet candidate transiting a star that had been considered a possible member of NGC 1817, potentially making it the first discovery of a giant transiting planet in an open cluster. Via follow-up observations and modeling, we were able to validate the candidate as a genuine transiting planet, but our analysis showed that the host star is not a cluster member. In Section 2, we describe our follow-up observations. In Section 3, we describe our analysis used to determine system parameters, validate the planet, and disqualify the star as a cluster member. In Section 4, we discuss the implications of our findings and methods for future planet searches in clusters and conclude. 

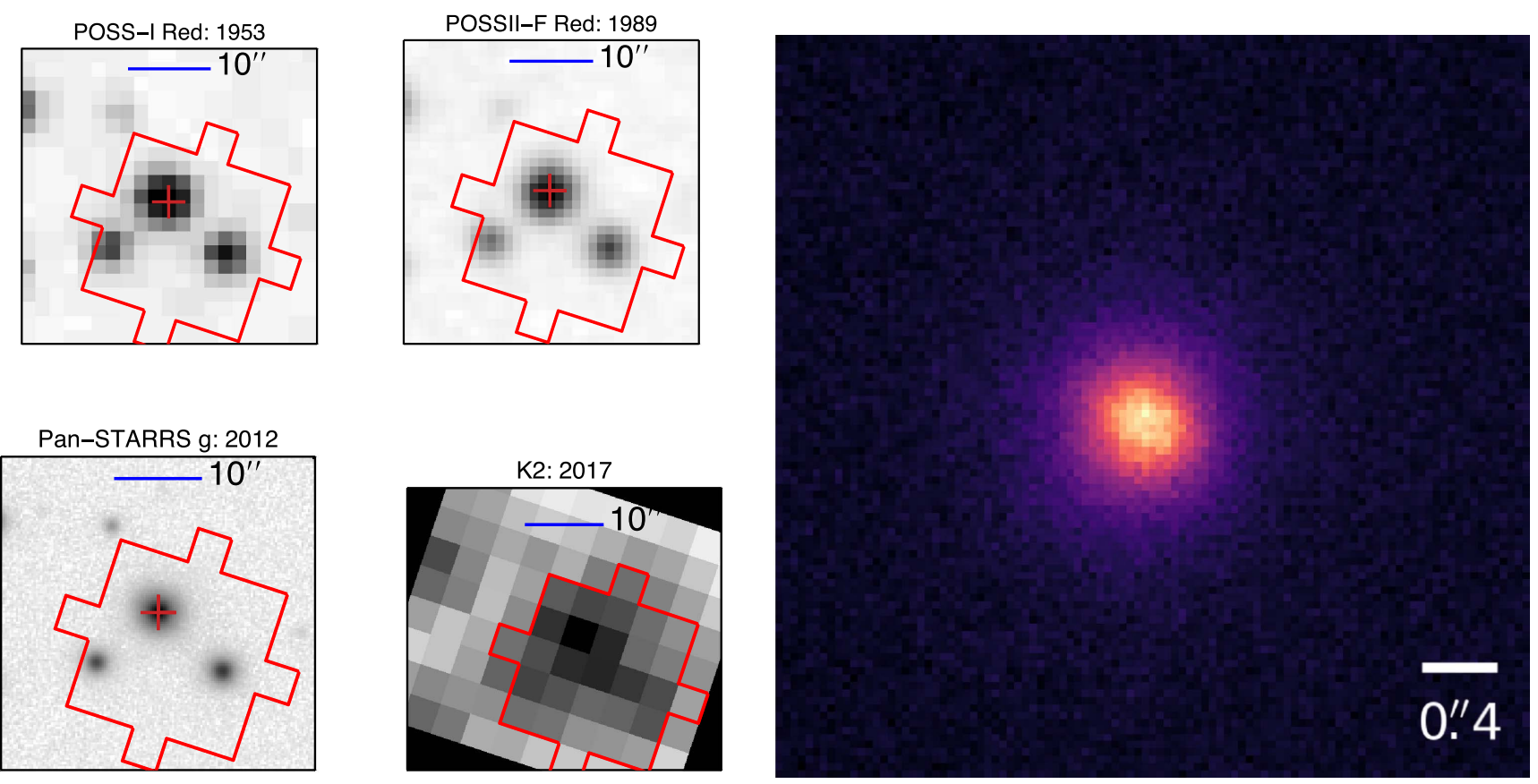

Figure 1. Top left: image from the first Palomar Sky Survey (POSS-I) with the red outline indicating the shape of the aperture chosen for reduction. Top middle: image from the second Palomar Sky Survey (POSSII). Bottom left: image from Panoramic Survey Telescope and Rapid Response System (PanSTARRS). Bottom middle: image from $K 2$ field of view rotated to match the orientation of the previous three images. Right: high-resolution image of the target obtained with Robo-AO.

\section{Observations}

\subsection{K2 Light Curve and Archival Imaging}

K2-308 (also known as EPIC 246865365) was observed from 2017 March 8 to May 27 by Kepler in the thirteenth campaign of its $K 2$ mission. We downloaded the subsequent calibrated target pixel files (TPFs) and extracted the light curve from the TPF using an aperture of about $20^{\prime \prime}$ in diameter. The light curve was processed following Vanderburg \& Johnson (2014) to produce a photometric light curve free of systematic effects due to the instrument's unstable pointing. We then performed a transit search using a box-least-squares periodogram search (Kovács et al. 2002; Vanderburg et al. 2016), and the light curve was visually inspected to ascertain with high confidence that the detected events were consistent with real planetary transits. We note that there was no obvious stellar rotation signal in the light curve. We then rederived the $K 2$ systematics correction by simultaneously fitting for the transits, $K 2$ roll systematics, and long-term trends in the data set, following Vanderburg et al. (2016). We use the light curve with this better-optimized systematics correction in our analysis throughout the rest of the paper.

Kepler has large $\left(4^{\prime \prime}\right)$ pixels and a $6^{\prime \prime}$ undersampled pointspread function, so we downloaded higher resolution archival images of the region of sky around K2-308 from the first and second Palomar Observatory Sky Surveys and the PanSTARRS survey. These images reveal that there are three stars within the photometric aperture that we used to extract the $K 2$ light curve (K2-308 and two other, slightly fainter stars), any one of which could plausibly be the source of the transit signals (see Figure 1). We later used seeing-limited follow-up photometry to determine the host (described in Section 2.2).

\subsection{Seeing-limited Follow-up Photometry}

In order to determine which star hosted the signal, we performed seeing-limited follow-up photometry. Seeing-limited photometry can prove useful when needing to rule out nearby eclipsing binaries as sources of the transit since many ground-based telescopes can achieve higher spatial resolution than focus-limited telescopes like Kepler. We observed the predicted transit that occurred on UT 2017 November 16 using the $1.2 \mathrm{~m}$ telescope at the Fred Lawrence Whipple Observatory (FLWO) on Mount Hopkins, Arizona. Images were taken using KeplerCam, a wide-field CCD camera with a 23!1 square field of view and resolution of 0 !" 336 per pixel. The target was observed using the Sloan $i$-band filter with an exposure time of 180 seconds.

Observing conditions were nominal with a measured seeing of 1"!98 (FWHM). Observations of the target began 20 minutes before predicted ingress and ended $2 \mathrm{hr}$ after the predicted egress with an average airmass of 1.18. As a result, observations during the entire $3.2 \mathrm{hr}$ predicted transit duration were obtained. Since KeplerCam is a single-chip CCD readout by 4 amps, the raw images are saved in four sections that we later stitched together with an IDL script. Standard IDL routines were also used to calibrate the images (Carter et al. 2011).

Differential aperture photometry was performed using AstroImageJ (AIJ; Collins et al. 2017). The AIJ apertures are made up of three concentric circular rings (with sizes chosen based on the measured FWHM seeing for the target) defining three separate regions: an inner circular aperture for measuring the star's flux (with radius $r<6^{\prime \prime}$, an annulus acting as a buffer between the inner and outer apertures; $6^{\prime \prime}<r<18^{\prime \prime}$ ), and an outer annulus measuring the sky background $\left(18^{\prime \prime}<r<25^{\prime \prime}\right)$. In order to determine the source of the transit signal, relative 
fluxes were measured for the target star, any neighboring stars suspected to contaminate the signal from the target, and comparison stars for the target star and potential contaminant stars. $\mathrm{BJD}_{\mathrm{TDB}}$ timestamps were used in order to remain consistent with data from Kepler.

We detected a transit signal consistent in transit depth and timing with the $K 2$ light curve from the target star while the neighboring stars remained flat throughout the night, confirming the target as the host.

\subsection{Spectroscopy}

\subsubsection{Tull Spectrograph at McDonald Observatory}

We observed K2-308 using the high-resolution crossdispersed echelle spectrometer on the Harlan J. Smith $2.7 \mathrm{~m}$ telescope at McDonald Observatory (Tull et al. 1995). This spectrometer is fed by a 1 ." $2 \times 8$ ". 2 slit and has wavelength coverage from 375 to $1020 \mathrm{~nm}$. These spectra were obtained on 2018 January 28, 29 and February 25 with a resolving power of $\Delta \lambda / \lambda \approx 60,000$. Exposure times of 3600 s yielded signal-tonoise ratios $(\mathrm{S} / \mathrm{Ns})$ of about 25 per resolution element at $565 \mathrm{~nm}$.

For each observation, three successive short exposures were taken to remove the energetic particle collisions on the detector. In order to obtain an accurate flux-weighted barycentric correction, an exposure meter was used. The raw data were then processed using the Image Reduction and Analysis Facility (IRAF) routines for bias subtraction, order extraction, and flat fielding. For each spectral order, apertures were traced and an extraction algorithm was applied to retrieve the stellar flux as a function of wavelength.

To calibrate wavelength and remove spectrograph drifting, we obtained bracketing exposures of a $\mathrm{Th}-\mathrm{Ar}$ hollow cathode lamp, which enabled calculation of absolute RVs from the spectra. We calculate an absolute $\mathrm{RV}$ of $\approx 9 \mathrm{~km} \mathrm{~s}^{-1}$ for this star and calculate stellar parameters from the spectra using Kea (Endl \& Cochran 2016), as described in Section 3.2.

\subsubsection{Tillinghast Reflector Echelle Spectrograph at Whipple Observatory}

We also observed K2-308 on 2018 January 22 and February 6 using the Tillinghast Reflector Echelle Spectrograph (TRES) on the $1.5 \mathrm{~m}$ telescope at FLWO. The spectra were acquired at a spectral resolving power of $\lambda / \Delta \lambda=44,000$ in a series of three exposures, each with an exposure time of $1200 \mathrm{~s}$. Between these exposures, bracketing exposures of a Th-Ar hollow cathode lamp were taken. However, due to the target's $V$-magnitude of 15 , the obtained spectra only yielded $\mathrm{S} / \mathrm{Ns}$ of 12 per resolution element at $518 \mathrm{~nm}$ even in good observing conditions. These spectra were too weak to use to derive accurate stellar parameters for the system or to derive RVs with our standard procedure, which uses the strongest observed spectrum of a star as the template for cross-correlation. Instead, we use a similar star from Praesepe as the template to derive RVs for the system; this process is described further in Section 3.3.

\subsection{Adaptive Optics Imaging}

We obtained images of K2-308 (as seen in Figure 1) using Robo-AO at the Kitt Peak National Observatory $2.1 \mathrm{~m}$ telescope (Baranec et al. 2014; Jensen-Clem et al. 2018). Robo-AO is a robotic laser guide-star adaptive optics system that can be used to determine if there are other potential sources in the Kepler photometric apertures affecting the transit signal (e.g., Law et al. 2014).

Observations were acquired using a long-pass filter that cuts at $\lambda=600 \mathrm{~nm}$ on UT 2018 March 3 as a series of frametransfer exposures at a rate of 8.6 frames per second for a total time of $120 \mathrm{~s}$. Effective seeing at the time of observation was measured to be approximately $1 . " 6$ in a $10 \mathrm{~s}$ exposure captured during the setup of the adaptive optics system. Because of the poor seeing and faintness of the target, we relied on the faintstar shift-and-add pipeline, described by Jensen-Clem et al. (2018), to combine the short exposures to maximize the $\mathrm{S} / \mathrm{N}$ of the final processed image. The final image width of K2-308 was measured to be 0.43 , and, from visual inspection, it appears there are no neighboring stars $\lesssim 2$ magnitudes fainter than K2-308 close enough to contaminate its signal.

\section{Analysis \\ 3.1. Transit Light Curve}

Due to the three stars in the photometric aperture (Figure 1), before measuring transit parameters, we applied a dilution correction to the $K 2$ light curve. Assuming the flux contained within the aperture is solely from those three stars, we obtained the Gaia $G$-magnitudes (comparable to the Kepler band) for the three stars within the aperture and converted them to fluxes. After confirming that the target was indeed the host of the transit signal (see Section 2.2), we determined the fraction of the flux within the aperture coming from the target star and applied the corresponding dilution,

$$
F_{\text {new }}=\frac{F-(1-d)}{d},
$$

where $F_{\text {new }}$ is the corrected flux, $F$ is the original flux, and $d$ is the fraction of the aperture's flux coming from the target star. For the target, we found $d=0.731$.

We also calculated the dilution using Kepler's point-spread function to model the flux fraction in the aperture and find $d=0.751$. This results in $\approx 3 \%$ change in transit depth compared to our initial calculation. However, we find an uncertainty of $\approx 10 \%$ for the transit depth in Section 3.1 indicating that this difference in dilution does not significantly change our results.

We measured transit parameters for the system by fitting the $K 2$ light curve (flattened by dividing away the best-fit lowfrequency variability from the simultaneous transit/systematics/low-frequency fit) and KeplerCam light curve with a Markov Chain Monte Carlo (MCMC) algorithm, emcee (Foreman-Mackey et al. 2013), using model light curves produced by the batman package (Kreidberg 2015). This model follows the Mandel \& Agol (2002) algorithm and was oversampled and binned to match Kepler's long exposure times as done by Kipping (2010). We also oversampled and binned the model used for the KeplerCam data to account for the 180 second exposure time.

Our model parameters include the planet-to-star radius ratio $\left(R_{p} / R_{*}\right)$, epoch of the first $K 2$ transit mid-point $\left(t_{0}\right)$, orbital period, scaled semimajor axis $\left(a / R_{*}\right)$, and orbital inclination. We also fit for normalization coefficients $\left(C_{1}, C_{2}\right)$ for the KeplerCam transit so that both the $K 2$ and KeplerCam data could be used together for measuring transit parameters. We introduce $C_{1}$ and $C_{2}$ in the following manner to fit for the 

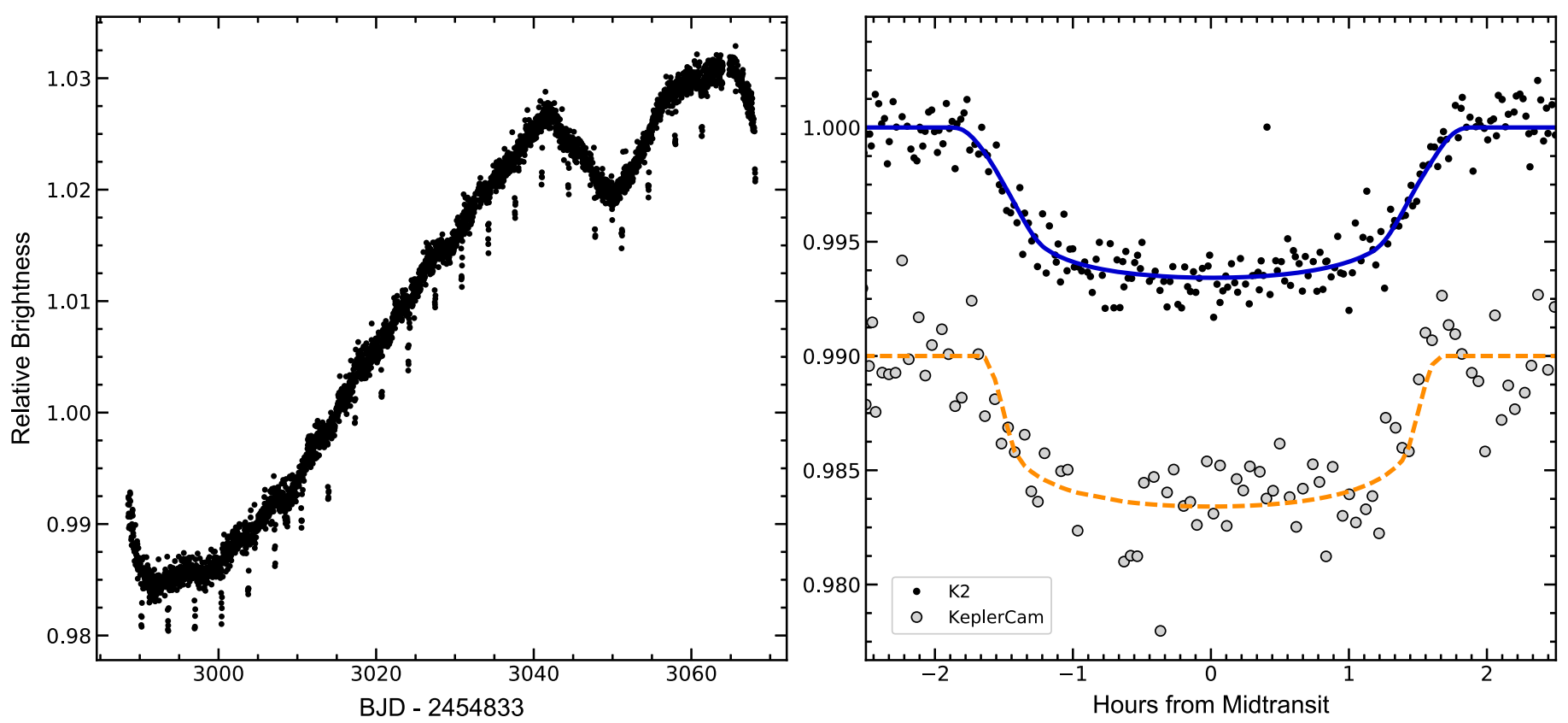

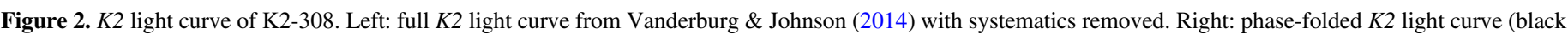

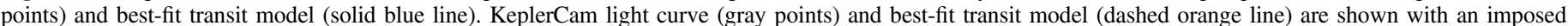
relative offset of 0.01 for clarity.

offset:

$$
\Theta_{\mathrm{kepcam}}=\theta_{\mathrm{kepcam}}\left(C_{1}+C_{2}\left(\frac{t-t_{0}}{t_{\mathrm{tot}}}\right)\right)
$$

where $\Theta_{\text {kepcam }}$ is the full forward modeled light curve, $\theta_{\text {kepcam }}$ is the light curve model generated by BATMAN, $C_{1}$ and $C_{2}$ are the flux offset and slope, $t$ is time of each KeplerCam flux measurement, $t_{0}$ is the first KeplerCam timestamp, and $t_{\text {tot }}$ is the total duration of the KeplerCam observations.

Assuming a quadratic limb-darkening law, we impose Gaussian priors on the two limb-darkening coefficients, $u_{1}$ and $u_{2}$, centered on 0.324 and 0.299 following Claret \& Bloemen (2011). We run the sampling with a fixed eccentricity of zero and a fixed longitude of periastron of $90^{\circ}$.

We sampled the parameter space with 150 walkers for 50,000 steps and discarded the first 5000 as burn-in. The transit light curves and best-fit model are shown in Figure 2, and transit parameters and uncertainties are listed in Table 1; for each parameter, we report the median value with errors as the 16 th and 84th percentiles corresponding to $1 \sigma$ errors for a Gaussian distribution.

\subsection{Stellar Parameters}

We were able to measure spectroscopic parameters of K2-308 by summing the three McDonald spectra and analyzing the coadded spectrum with the Kea software package. Kea compares the input spectrum with a large grid of synthetic model stellar spectra to determine spectroscopic properties including stellar effective temperatures, surface gravities, and metallicities (Endl $\&$ Cochran 2016). From the Kea analysis, we find a $T_{\text {eff }}$ of $6100 \pm 263 \mathrm{~K}$, metallicity of $[\mathrm{Fe} / \mathrm{H}]=-0.10 \pm 0.08 \mathrm{dex}$, surface gravity of $\log g_{\text {cgs, Kea }}=3.94 \pm 0.44 \mathrm{dex}$, and a projected rotational velocity with an upper limit of $v \sin i \leqslant$ $29 \pm 5 \mathrm{~km} \mathrm{~s}^{-1}$.
Assuming a circular orbit and using the $K 2$ transit photometry, we estimate a stellar density with the measured $a / R_{*}$, period, and Kepler's third law following Seager \& Mallén-Ornelas (2003). We assumed a zero eccentricity for the system since a majority of short-period planets, including K2$308 \mathrm{~b}$, have circularization timescales much less than the age of their host stars. ${ }^{9}$

With the stellar density and measured spectral parameters including metallicity, effective temperature, and surface gravity, we then determine the mass, radius, and expected parallax of the star following Vanderburg et al. (2017). This was done using an MCMC with an affine invariant ensemble sampler to explore parameter space in stellar mass, metallicity, and age based on the Yonsei-Yale isochrones (Yi et al. 2001), while imposing priors on the stellar density, metallicity, temperature, and surface gravity from our previous analysis.

We find that K2-308 has a mass of $1.09 \pm 0.09 M_{\odot}$ and a radius of $1.24 \pm 0.10 R_{\odot}$, indicating a late F-type dwarf star. Based on the models and $V$-band brightness of K2-308, we calculate an expected parallax of $0.66 \pm 0.09$ mas. Our predicted parallax is within $2.5 \sigma$ of the Gaia DR2 value $(-1.25 \pm 0.72$ mas $)$, a nondetection due to the star's faintness and distance.

\subsection{Radial Velocity Analysis}

We measured the absolute RV of K2-308 from the McDonald spectra using Kea. Using observations of RV standard stars taken on the same night, we place the RVs on the IAU absolute velocity scale.

\footnotetext{
9 We confirmed that the circularization timescale of K2-308b is much shorter than the age of the star following Goldreich \& Soter (1966) and Mills \& Fabrycky (2017). Using the 50th percentile of the calculated mass limit distribution (described later in Section 3.3), we estimate a conservative upper limit on the circularization timescale of $315 \mathrm{Myr}$. More reasonable estimates for the planetary mass, assuming it is similar to Saturn, give circularization timescales an order of magnitude shorter, much less than the age of the host star.
} 
Table 1

System Parameters for K2-308

\begin{tabular}{|c|c|c|c|}
\hline & & $68.3 \%$ & \\
\hline Parameter & Value & $\begin{array}{c}\text { Confidence } \\
\text { Interval } \\
\text { Width }\end{array}$ & Comment \\
\hline
\end{tabular}

Other Designations

Gaia DR2

3393982701757444352

2MASS J05132138

$+1624510$

WISE J051321.40

$+162451.1$

EPIC 246865365

Basic Information

Orbital inclination, $i$ [deg]
R.A.

decl.

Proper motion in R.A. [mas $\mathrm{yr}^{-1}$ ]

Proper motion in decl. [mas $\mathrm{yr}^{-1}$ ]

Absolute RV [ $\left.\mathrm{km} \mathrm{s}^{-1}\right]$

$V$-magnitude

$K$-magnitude

Kepler-band $K p$

magnitude

\section{Stellar Parameters}

Mass $M_{\star}\left[M_{\odot}\right]$

Radius $R_{\star}\left[R_{\odot}\right]$

Limb-darkening $u_{1}$

Limb-darkening $u_{2}$

$\log g_{\text {Kea }}$ [cgs]

Metallicity $[\mathrm{Fe} / \mathrm{H}]$

$T_{\text {eff }}$ [K]

\section{K2-308b}

Orbital period, $P$ [days]

Radius ratio, $R_{P} / R_{\star}$

Scaled semimajor axis, $a / R_{\star}$

Transit impact para-

meter, $b$

Transit duration, $t_{14}[\mathrm{hr}]$

Time of transit $t_{t}$ [BJD]

Planet radius $R_{P}\left[R_{\oplus}\right]$

Planet radius $R_{P}\left[R_{\text {Jupiter }}\right]$

$05: 13: 21.39$
$+16: 24: 51.13$
-2.54
2.94
8.97
14.96
13.20
14.75

$\begin{array}{ccc} & & \mathrm{A} \\ & & \mathrm{A} \\ \pm & 1.19 & \mathrm{~A} \\ \pm & 0.88 & \mathrm{~A} \\ \pm & 1.79 & \mathrm{~B} \\ \pm & 0.04 & \mathrm{~A} \\ \pm & 0.02 & \mathrm{~A} \\ & & \mathrm{~A}\end{array}$

Note. A: parameters come from the EPIC catalog (Huber et al. 2016) and Gaia Data Release 2 (Gaia Collaboration et al. 2018); B: parameters come from analysis of the two TRES spectra and three McDonald spectra (Section 3.3); C: parameters come from analysis of McDonald spectra using Kea (Section 3.2); D: parameters come from the analysis of the $K 2$ and KeplerCam light curves (Section 3.1); and E: Gaussian priors of imposed on $u_{1}$ and $u_{2}$ centered on 0.324 and 0.299, respectively, with width 0.1 from Claret \& Bloemen (2011).

Because the TRES observations were too weak for us to derive reliable RVs with our standard procedures (crosscorrelating against a suite of model spectra), we performed a custom analysis. We cross-correlated the two TRES spectra against a high S/N TRES spectrum of the Praesepe star EPIC $211926132\left(T_{\text {eff }}=6250 \mathrm{~K} ; v \sin i=10 \mathrm{~km} \mathrm{~s}^{-1}\right)$ and estimated the relative RV of EPIC 211926132 using multiple echelle orders. We measured an $\approx 350 \mathrm{~m} \mathrm{~s}^{-1}$ shift between the two spectra, with uncertainties of $300 \mathrm{~m} \mathrm{~s}^{-1}$ velocity. We placed the two TRES velocities on the IAU scale using historical observations of RV standard stars. There were no significant variations when combined with the absolute RVs derived from the McDonald spectra as seen in Figure 3.

We calculated the mass limit of the planet candidate using the RVs from both McDonald and TRES, which we show in Figure 3. This was done by phase-folding the RV observations using the measured period from the transit fitting (Section 3.1) and fitting a sine curve with emcee to determine the semiamplitude of the system. Following Cumming et al. (1999), we determined the planet mass limit from the semi-amplitude. Using the calculated stellar mass (described in Section 3.2) and the distribution of semi-amplitudes calculated by the emcee fit, we calculate a posterior probability distribution for the planet's mass.

Our analysis shows that if the transiting object orbits K2308 , its mass is much less than that of a stellar companion. While the use of another star as the template can introduce small systematic errors to the velocities, we can confidently exclude the presence of an eclipsing binary orbiting the host star.

We note that despite obtaining weaker spectra from TRES compared to the spectra from McDonald, we derive absolute RVs with smaller uncertainties. This can be attributed to the better instrumental stability and fiber-fed setup of TRES compared to the slit-fed Tull spectrograph at McDonald.

\section{4. (Non-)Membership in NGC 1817}

NGC 1817 is an open cluster similar in age to the Hyades located $(\approx 1 \mathrm{Gyr})$ in the constellation of Taurus. Prior to Gaia Data Release 2 (DR2), its cluster members were reported to have a proper motion of $(3.56,-6.7)$ mas $\mathrm{yr}^{-1}$ in (R.A., decl.), and an RV of $65.3 \pm 0.1 \mathrm{~km} \mathrm{~s}^{-1}$ (Balaguer-Núñez et al. 2004; Wu et al. 2009). Recently, the cluster's proper motion was updated using data from Gaia DR2, giving $(0.485,-0.89)$ mas $\mathrm{yr}^{-1}$ in (R.A., decl.) (Cantat-Gaudin et al. 2018).

K2-308 was long considered to be a possible member of the cluster given its similar proper motion of $(3.3 \pm 4,-1.2 \pm 4)$ mas $\mathrm{yr}^{-1}$ in (R.A., decl.) (Krone-Martins et al. 2010). In fact, Krone-Martins et al. (2010) calculated the star to have a 92.1\% membership probability.

However, the reported Gaia DR2 proper motion of

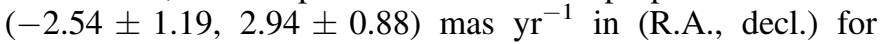
this star suggests that the star is not associated with the cluster as shown in Figure 4. Additionally, our TRES and McDonald spectra indicate an absolute $\mathrm{RV}$ of $9 \mathrm{~km} \mathrm{~s}^{-1}$, strongly indicating that K2-308 is unassociated with NGC 1817.

\subsection{False-positive Probability}

It is important to acknowledge that the source of the transit could be due to other astrophysical sources. This is especially true in crowded fields such as this one, where there are multiple stars within the $K 2$ aperture. This conundrum motivated our follow-up observations, which we have described throughout the paper. Here, we summarize the observations and describe the validation tests we use to show that with high likelihood, K2-308 is indeed a planet-star system. 

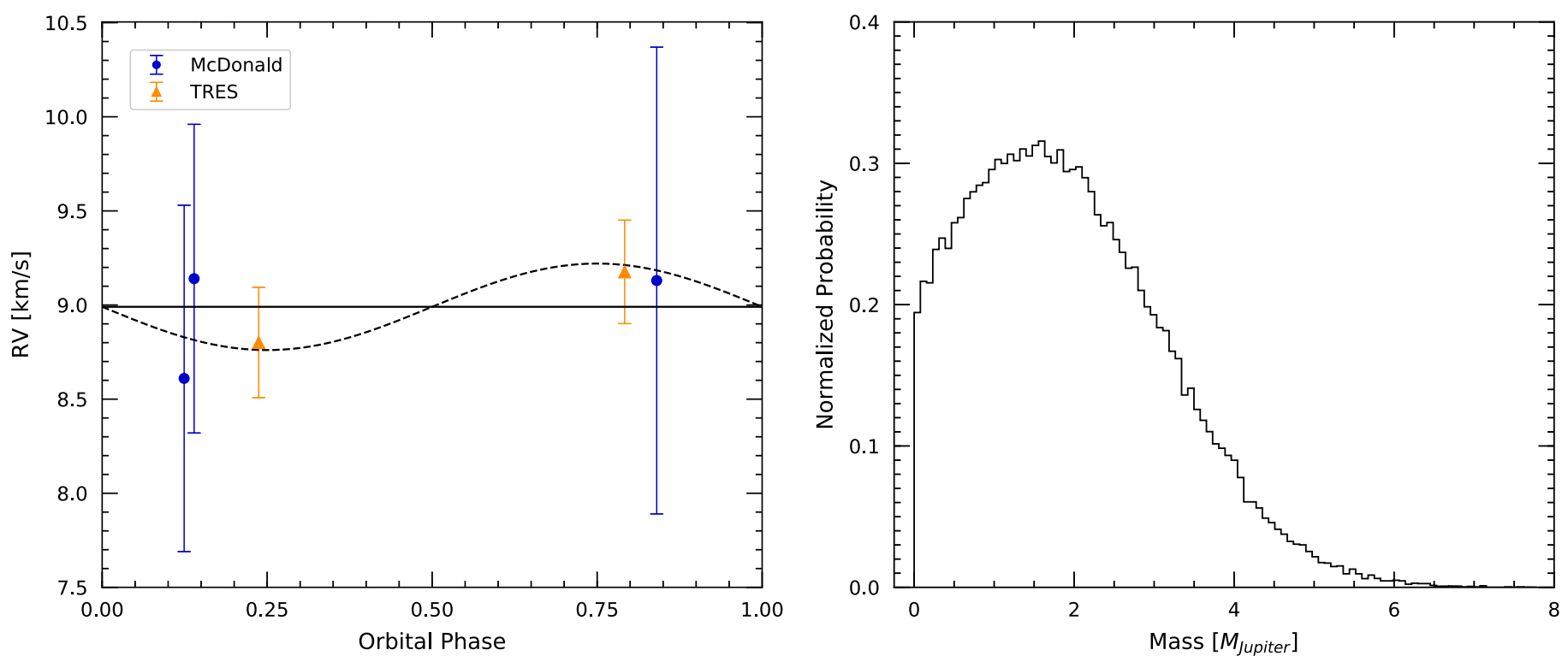

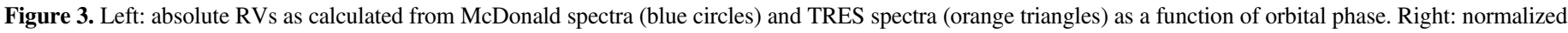

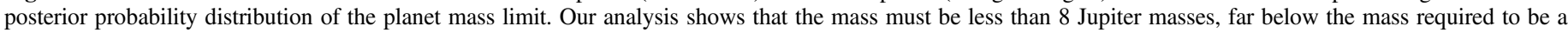
stellar companion.

From the seeing-limited photometry results, we were able to determine that the signal was indeed coming from the target, eliminating the other two stars in the aperture as potential contaminants (Figure 2). Based on our analysis with the TRES and McDonald spectra, the RVs rule out the scenario in which the target is an eclipsing binary system (Figure 3 ). Robo-AO results also reveal that there are no neighboring stars $\leqslant \Delta 2$ mag close enough to the target to contaminate its signal (Figure 1). However, this does not necessarily rule out all cases of potential nearby star contamination, so we statistically validate the system as well to affirm that K2-308 is the source of the transits. We calculate the false-positive probability (FPP) of K2-308b using the Validation of Exoplanet Signals using a Probabilistic Algorithm (VESPA) software package (Morton 2015), an open-source implementation of the method developed by Morton (2012). Given inputs like the transit shape, orbital period, host stellar parameters, and other observational constraints, VESPA calculates the FPP of transiting planet candidates by considering potential falsepositive scenarios such as blends with hierarchical (physically associated) eclipsing binary systems and coincidentally aligned background eclipsing binary systems. After imposing our constraints on the system, VESPA returned an FPP of $0.01 \%$. We therefore consider the planet candidate statistically validated.

\section{Discussion}

In this work, we have shown that there is a hot Saturn orbiting K2-308. This star was long thought to be a member of the open cluster NGC 1817 due to similar proper motions and positions. However, when we conducted follow-up observations, we found that while the planet is likely real, the star is likely not a member of the cluster. Prior to the release of Gaia DR2, we found the star was not a member due to the RVs we measured $\left(\approx 9 \mathrm{~km} \mathrm{~s}^{-1}\right)$ compared to those reported for the cluster $\left(65.3 \mathrm{~km} \mathrm{~s}^{-1}\right)$. With the release of Gaia DR2, we have better measured kinematics for both K2-308 and NGC 1817. We found that the star and cluster do not have common RVs or proper motions despite what was previously reported, confirming our findings that this star was not a cluster member.

This work demonstrates the important role Gaia will play in the search for planets in clusters. While we believe that the known cluster planets do indeed orbit cluster members (since follow-up of the planets included RV measurements confirming membership), the full sample of potential planet hosts in clusters still remains to be established. With Gaia, we will improve cluster membership probabilities allowing us to create a more accurate and robust sample of cluster stars to search. This, in turn, will improve cluster planet occurrence rates. Additionally, these new membership lists would give us the opportunity to determine if previously unidentified cluster members had planets discovered around them serendipitously in earlier planet hunting surveys.

One of the key elements of our follow-up observations was seeing-limited imaging. When initially performing photometry on the $K 2$ data, there were three stars within the aperture leaving the transit host unknown. Using KeplerCam, we performed seeing-limited photometry to successfully determine the target as the host star and eliminate potential contaminant sources. We expect a similar problem with TESS as the cameras' large pixels will also result in multiple stars within an aperture (Ricker et al. 2015). Seeing-limited follow-up is already being used with TESS (e.g., Vanderspek et al. 2019; Günther et al. 2019), but the challenge will be even greater in cluster regions. For example, about 2-4 stars from NGC 1817 would be found in just one TESS pixel.

The fact that K2-308 is not a member of NGC 1817 is another null result in the search for transiting giant planets in clusters. Despite the many attempted targeted surveys in the past to detect such planet systems in various open clusters (e.g., Gilliland et al. 2000; Pepper \& Gaudi 2005; Aigrain et al. 2007; Beatty \& Gaudi 2008), a successful transit detection of a giant planet in a cluster remains elusive. This has be attributed to the intrinsically low occurrence rate of giant planets and a paucity of stars in open clusters, leading to a low probability of a detection (van Saders \& Gaudi 2011). TESS will be 

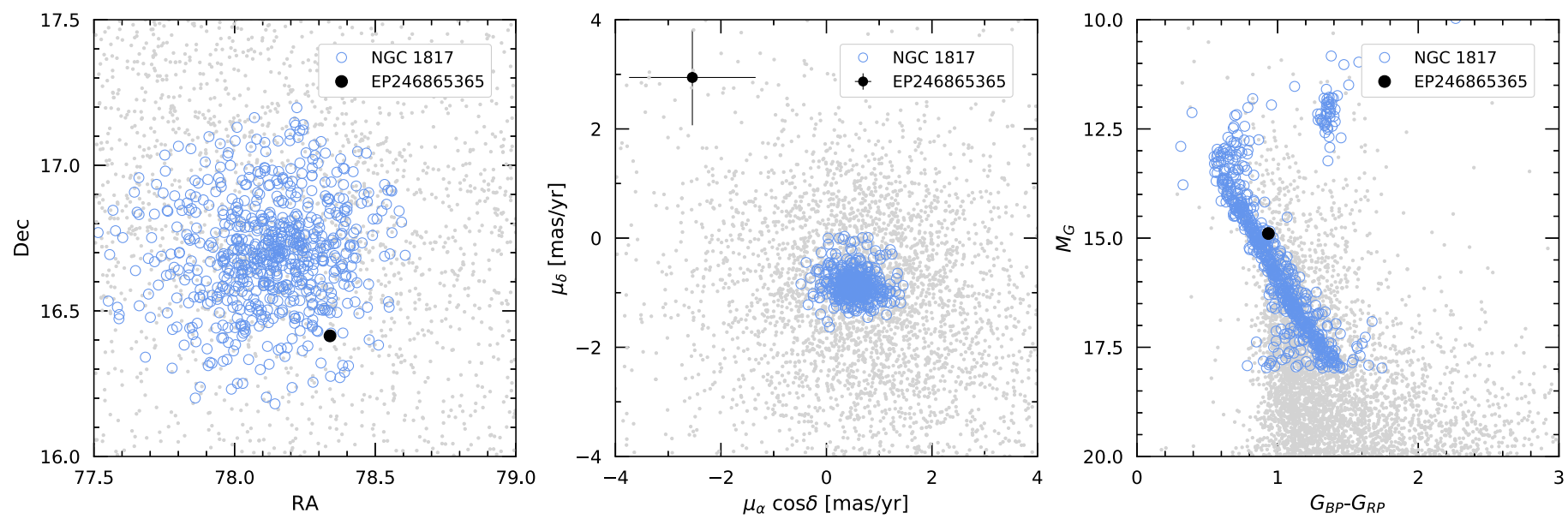

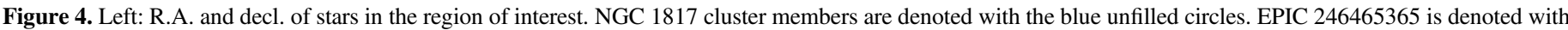

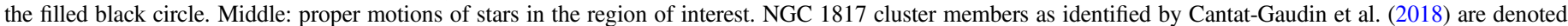

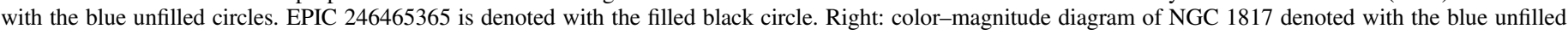
circles as identified by Cantat-Gaudin et al. (2018) and EPIC 246465365 is denoted with the filled black circle.

instrumental in continuing this search as it will observe a number of clusters and will be sensitive to such giant planets. With new membership information from Gaia and new data from TESS, perhaps the first transiting giant planet in an open cluster will soon be found.

We thank the anonymous referee for a quick and constructive review.

R.R. gratefully acknowledges the support of the Columbia University Bridge to the Ph.D. Program in STEM. The work of A.V. was performed in part under contract with the California Institute of Technology/Jet Propulsion Laboratory funded by NASA through the Sagan Fellowship Program executed by the NASA Exoplanet Science Institute. C.B. acknowledges support from the Alfred P. Sloan Foundation. D.W.L. acknowledges partial support from the TESS mission through a sub-award from the Massachusetts Institute of Technology to the Smithsonian Astrophysical Observatory (SAO) and from the Kepler mission under NASA Cooperative agreement NNX13AB58A with SAO.

This research has made use of NASA's Astrophysics Data System and the NASA Exoplanet Archive, which is operated by the California Institute of Technology, under contract with the National Aeronautics and Space Administration under the Exoplanet Exploration Program. The National Geographic Society-Palomar Observatory Sky Atlas (POSS-I) was made by the California Institute of Technology with grants from the National Geographic Society. The Oschin Schmidt Telescope is operated by the California Institute of Technology and Palomar Observatory.

This paper includes data collected by the Kepler mission. Funding for the Kepler mission is provided by the NASA Science Mission directorate. Some of the data presented in this paper were obtained from the Mikulski Archive for Space Telescopes (MAST). STScI is operated by the Association of Universities for Research in Astronomy, Inc., under NASA contract NAS5-26555. Support for MAST for non-HST data is provided by the NASA Office of Space Science via grant NNX13AC07G and by other grants and contracts. This work has made use of data from the European Space Agency (ESA) mission Gaia (https://www.cosmos.esa.int/gaia), processed by the Gaia Data Processing and Analysis Consortium (DPAC; https://www.cosmos.esa.int/web/gaia/dpac/consortium). Funding for the DPAC has been provided by national institutions, in particular the institutions participating in the Gaia Multilateral Agreement.

The Robo-AO instrument was developed with support from the National Science Foundation under grants AST-0906060, AST-0960343, and AST-1207891, IUCAA, the Mt. Cuba Astronomical Foundation, and by a gift from Samuel Oschin. The Robo-AO team thanks NSF and NOAO for making the Kitt Peak $2.1 \mathrm{~m}$ telescope available. We thank the observatory staff at Kitt Peak for their efforts to assist Robo-AO KP operations. Robo-AO KP is a partnership between the California Institute of Technology, the University of Hawai' $i$, the University of North Carolina at Chapel Hill, the InterUniversity Centre for Astronomy and Astrophysics (IUCAA) at Pune, India, and the National Central University, Taiwan. The Murty family feels very happy to have added a small value to this important project. Robo-AO KP is also supported by grants from the John Templeton Foundation and the Mt. Cuba Astronomical Foundation.

Some data are based on observations at Kitt Peak National Observatory, National Optical Astronomy Observatory (NOAO Prop. ID: 15B-3001), which is operated by the Association of Universities for Research in Astronomy (AURA) under cooperative agreement with the National Science Foundation. We are honored to be permitted to conduct observations on Iolkam Duag (Kitt Peak), a mountain within the Tohono O'odham Nation with particular significance to the Tohono O'odham people.

Facilities: Kepler/K2, FLWO:1.5 m (TRES), FLWO:1.2 m (KeplerCam), KPNO2.1 m (Robo-AO), Smith (Tull), Gaia, Exoplanet Archive, MAST, CDS, ADS.

Software: AstroImageJ (Collins et al. 2017), astropy (Astropy Collaboration et al. 2013), batman (Kreidberg 2015), emcee (Foreman-Mackey et al. 2013), VESPA (Morton 2015).

\section{ORCID iDs}

Rayna Rampalli (10) https://orcid.org/0000-0001-7337-5936 Andrew Vanderburg (i) https://orcid.org/0000-0001-7246-5438 Allyson Bieryla (i) https://orcid.org/0000-0001-6637-5401 David W. Latham (1) https://orcid.org/0000-0001-9911-7388 Samuel N. Quinn (D) https://orcid.org/0000-0002-8964-8377 
Christoph Baranec (iD https://orcid.org/0000-0002-1917-9157

Michael L. Calkins (10 https://orcid.org/0000-0002-2830-5661

William D. Cochran (i) https://orcid.org/0000-0001-9662-3496

Dmitry A. Duev (iD https://orcid.org/0000-0001-5060-8733

Michael Endl (ib https://orcid.org/0000-0002-7714-6310

Rebecca Jensen-Clem (i) https://orcid.org/0000-0003-0054-2953

Nicholas M. Law (i) https://orcid.org/0000-0001-9380-6457

Andrew W. Mayo (ib https://orcid.org/0000-0002-7216-2135

Reed Riddle (iD https://orcid.org/0000-0002-0387-370X

Maïssa Salama (iD https://orcid.org/0000-0002-5082-6332

\section{References}

Aigrain, S., Hodgkin, S., Irwin, J., et al. 2007, MNRAS, 375, 29

Astropy Collaboration, Robitaille, T. P., Tollerud, E. J., et al. 2013, A\&A, 558, A33

Balaguer-Núñez, L., Jordi, C., Galadí-Enríquez, D., \& Zhao, J. L. 2004, A\&A, 426,819

Baranec, C., Riddle, R., Law, N. M., et al. 2014, ApJL, 790, L8

Beatty, T. G., \& Gaudi, B. S. 2008, ApJ, 686, 1302

Cantat-Gaudin, T., Jordi, C., Vallenari, A., et al. 2018, A\&A, 618, A93

Carter, J. A., Winn, J. N., Holman, M. J., et al. 2011, ApJ, 730, 82

Claret, A., \& Bloemen, S. 2011, A\&A, 529, A75

Collins, K. A., Kielkopf, J. F., Stassun, K. G., \& Hessman, F. V. 2017, AJ 153,77

Crossfield, I. J. M., Petigura, E., Schlieder, J. E., et al. 2015, ApJ, 804, 10

Cumming, A., Marcy, G. W., \& Butler, R. P. 1999, ApJ, 526, 890

Curtis, J. L., Vanderburg, A., Torres, G., et al. 2018, AJ, 155, 173

Donati, P., Beccari, G., Bragaglia, A., Cignoni, M., \& Tosi, M. 2014, MNRAS, 437, 1241

Endl, M., \& Cochran, W. D. 2016, PASP, 128, 094502

Foreman-Mackey, D., Hogg, D. W., Lang, D., \& Goodman, J. 2013, PASP, 125,306

Gaia Collaboration, Brown, A. G. A., Vallenari, A., et al. 2018, A\&A, 616, A1

Gilliland, R. L., Brown, T. M., Guhathakurta, P., et al. 2000, ApJL, 545, L47
Goldreich, P., \& Soter, S. 1966, Icar, 5, 375

Günther, M. N., Pozuelos, F. J., Dittmann, J. A., et al. 2019, arXiv:1903.06107

Howell, S. B., Sobeck, C., Haas, M., et al. 2014, PASP, 126, 398

Huber, D., Bryson, S. T., Haas, M. R., et al. 2016, ApJS, 224, 2

Jensen-Clem, R., Duev, D. A., Riddle, R., et al. 2018, AJ, 155, 32

Kipping, D. M. 2010, MNRAS, 408, 1758

Kovács, G., Zucker, S., \& Mazeh, T. 2002, A\&A, 391, 369

Kreidberg, L. 2015, PASP, 127, 1161

Krone-Martins, A., Soubiran, C., Ducourant, C., Teixeira, R., \& Le Campion, J. F. 2010, A\&A, 516, A3

Lammer, H., Stökl, A., Erkaev, N. V., et al. 2014, MNRAS, 439, 3225

Law, N. M., Morton, T., Baranec, C., et al. 2014, ApJ, 791, 35

Lopez, E. D., Fortney, J. J., \& Miller, N. 2012, ApJ, 761, 59

Mandel, K., \& Agol, E. 2002, ApJL, 580, L171

Mann, A. W., Gaidos, E., \& Gaudi, B. S. 2010, ApJ, 719, 1454

Mann, A. W., Gaidos, E., Mace, G. N., et al. 2016, ApJ, 818, 46

Meibom, S., Torres, G., Fressin, F., et al. 2013, Natur, 499, 55

Mills, S. M., \& Fabrycky, D. C. 2017, ApJL, 838, L11

Morton, T. D. 2012, ApJ, 761, 6

Morton, T. D. 2015, VESPA: False positive probabilities calculator, Astrophysics Source Code Library, ascl:1503.011

Pepper, J., \& Gaudi, B. S. 2005, ApJ, 631, 581

Pepper, J., Gillen, E., Parviainen, H., et al. 2017, AJ, 153, 177

Quinn, S. N., White, R. J., Latham, D. W., et al. 2012, ApJL, 756, L33

Quinn, S. N., White, R. J., Latham, D. W., et al. 2014, ApJ, 787, 27

Ricker, G. R., Winn, J. N., Vanderspek, R., et al. 2015, JATIS, 1, 014003

Sato, B., Izumiura, H., Toyota, E., et al. 2007, ApJ, 661, 527

Seager, S., \& Mallén-Ornelas, G. 2003, ApJ, 585, 1038

Tull, R. G., MacQueen, P. J., Sneden, C., \& Lambert, D. L. 1995, PASP, 107,251

van Saders, J. L., \& Gaudi, B. S. 2011, ApJ, 729, 63

Vanderburg, A., Becker, J. C., Buchhave, L. A., et al. 2017, AJ, 154, 237

Vanderburg, A., \& Johnson, J. A. 2014, PASP, 126, 948

Vanderburg, A., Latham, D. W., Buchhave, L. A., et al. 2016, ApJS, 222, 14

Vanderburg, A., Mann, A. W., Rizzuto, A., et al. 2018, AJ, 156, 46

Vanderspek, R., Huang, C. X., Vanderburg, A., et al. 2019, ApJL, 871, L24

Wu, Z.-Y., Zhou, X., Ma, J., \& Du, C.-H. 2009, MNRAS, 399, 2146

Yi, S., Demarque, P., Kim, Y.-C., et al. 2001, ApJS, 136, 417 